\title{
A Study on Analysis of Risk Groups for Post-Traumatic Stress Disorder Based on NEMA Research Data
}

\author{
JeongBeom Kim \\ Professor, Namseoul University, Korea \\ jbkim@nsu.ac.kr
}

\begin{abstract}
A typical mental problem that develops over a long period of time after experiencing a physically and mentally shocking event is known as post-traumatic stress disorder. Posttraumatic stress disorder is a complex psychological trauma that can lead to self-harm and self-harm and suicidal thoughts at risk, even in serious cases to suicide. The root cause of this phenomenon can be found in many places, but repeated trauma exposure and inadequate medical support in regard to the occupational specificity of fire fighters can be cited as the main cause. As such, the psychological post-traumatic stress disorder needs to be dealt with as a mental health problem in the public domain, beyond the mental health level of the individual. Even for stability, research on advancement of analysis tools for high-risk groups and preemptive prediction methods should be carried out along with the shift of awareness of the actual risk factors in modern society to encompass social problems in a broad sense. The purpose of this study is to analyze the risk group of post-traumatic stress disorders exposed to various types of trauma such as accidents, damages and disasters based on NEMA (National Emergency Management Association) data.
\end{abstract}

Keywords: PTSD (post-traumatic stress disorder), NEMA, Analysis, Cluster, Health Care

\section{Introduction}

The term Post-traumatic Stress Disorder (PTSD) was first used in the field of psychiatry through the 1980 American Diagnostic and Statistical Manual of Mental Disorders (DSMIII). PTSD began to attract attention through symptoms such as anxiety, sleep disorders, and depression caused by soldiers who were originally sent to war or conflict zones. It was used as a generic term. In particular, in the process of developing an advanced civilized society, PTSD high-risk groups are increasing day by day due to the enlargement and diversification of disasters, disasters, events, and accidents. While there is a clear difference in the intensity and response patterns of mental trauma that an individual accepts in a particular event, existing PTSD criteria have limitations in diagnosing these complex factors. Because it does not completely eliminate the possibility of contaminating the result data, the main purpose is to develop an analysis algorithm that can verify the reliability by developing an intelligent analysis tool based on NEMA (National Emergency Management Association) data.

\section{Related study}

Article history:

Received (January 19, 2020), Review Result (February 22, 2020), Accepted (April 6, 2020) 
The damage to mental health as well as physical trauma is on the rise due to social and natural disasters, and accidents that are diversifying and increasing day by day. In particular, in addition to direct and physical thinking experiences, indirect trauma exposures transmitted or contacted by family members or acquaintances can cause serious stress disorders. In particular, firefighters who carry out first aid and disaster prevention activities at various accident sites have a relatively high frequency and repetitive exposure to trauma compared to the general population. More than 30 firefighters have committed suicide in the past five years, suffering from post-traumatic stress disorder (PTSD), according to the National Emergency Management Agency (NEMA). NEMA conducted research last year from 32,212 firefighters. Of them, 4,462 or 13.9 percent of members fell in the category of those suffering PTSD. NEMA pledged to set up a plan last year to systematically help those with mental health problems and boost the welfare of fire-fighters. However, it has taken no effective steps as of yet. Among the firefighters who died in 2015, only two of them were on duty, while 12 were suicidal. The purpose of this study is to analyze the prevalence and actual status of PTSD by mental health damage factors of firefighters exposed to various types of trauma such as accidents and disasters. A typical mental problem that occurs over a long period of time after experiencing a physically and mentally shocking event is known as posttraumatic stress disorder (PTSD), which often comes with depression, sleep disorders, and panic disorder. PTSD is a complex psychological trauma that can lead to self-harm, and in some cases even to actual suicide. The root cause of this phenomenon can be found in many places, but the lack of repeated trauma exposure and adequate medical assistance can be attributed to the lack of occupational characteristics of firefighters [1].

As such, the psychological post-traumatic stress disorder of firefighters needs to be dealt with as a mental health problem in the public domain, beyond the mental health of individuals. Even for stability, research on advancement of analytical tools and high-prediction methods for PTSD high-risk groups should be conducted, along with a shift in the perception of risk factors in modern society. Also, based on the analyzed data, we will derive a classification system for PTSD pathogens and study how to use them as a key information asset for discovering risk factors, PTSD prevention, treatment and support programs. Fire officials have a higher risk of trauma than the general population, and they feel lethargy and depression over long periods of time when they witness or apprehend the dead bodies during public service. Even with these symptoms, professional psychological counseling and treatment support are not always in demand due to chronic fire shortage and lack of budget. In 2016, government fire department developed and distributed a web-based PTSD selfdiagnosis process to promote psychological diagnosis and mental health welfare of firefighters. This program was developed and distributed on the basis of psychological (psychiatric) medical examination through the system of "special health check-up" in Article 27 of the Health and Safety Management Regulation, but there is concern that the results of self-diagnosis will adversely affect his/her personnel in the future. Because of the high rate of inaccurate responses, the reliability of the results was deteriorated. If we use RNN (Recurrent Neural Network) analysis, research data can be analyzed in statement type. Below program code and figure show the example of using Keras and RNN analysis [2].

The first phase is to creation of data set. In this phase, following activities are included.

Import original data or generate data through simulation. Create training set, and verify data set. Check format conversion is performed to train and evaluate the deep learning model.

The second phase is to configure model. In this phase, following activities are included.

After creating the sequence model, add and configure the necessary layers. When you need a more complex model, use the Keras function API. The third phase is to set up model 
training process. In this phase, following activities are included. Set up for learning before learning. Define the loss function and optimization method. Keras uses the compile() function. The fourth phase is to train model. In this phase, following activities are included. Train with a model constructed using a training set. In Keras, we use the fit() function. The fifth phase is to check training process. In this phase, following activities are included. When training the model, we measure the loss and accuracy of the training set and verification set. Determine the learning situation by looking at the loss and accuracy trend according to the number of repetitions. The sixth phase is to verify model. In this phase, following activities are included. Evaluate the trained model with the prepared test set. Keras uses the evaluate() function. The seventh phase is to use model finally. In this phase, following activities are included. Get the output of the model with random inputs. Keras uses the predict() function [3][4][5][6][7].

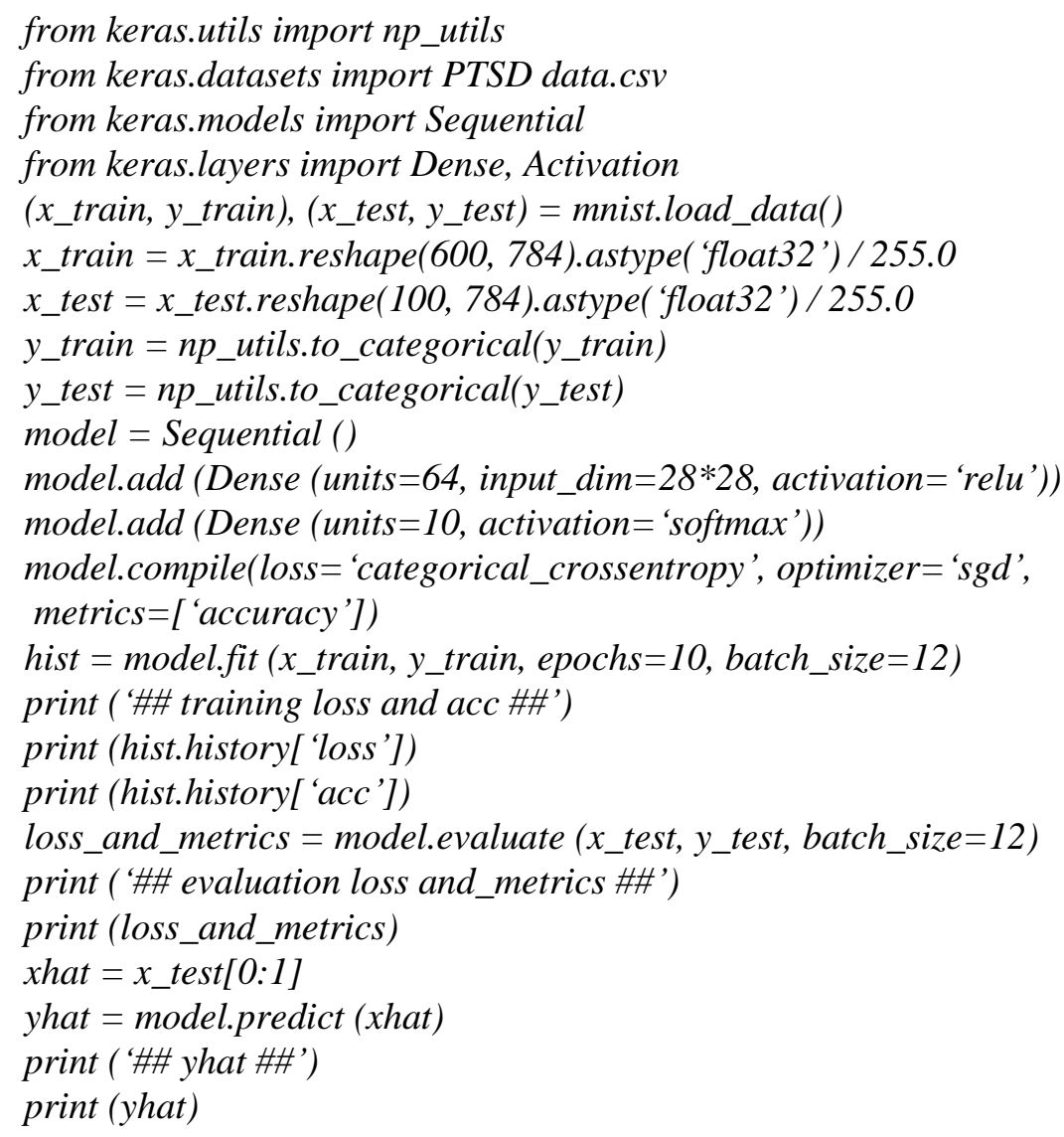

\section{Analysis result}

The purpose of this study is to analyze the research data to express early warning of posttraumatic stress disorder by mapping the report data to the 119-situation room. The firefighting field activity report data of the crews are the main source of research data. Based on the question and answer method, there is a variable that the reliability of the result will be deteriorated if the individual firefighters are involved. By analyzing the log of emergency activities, this paper aims to secure the correlation, objectivity and reliability of the results. Fire officials who are responsible for the property and safety of the people are generally occupied with the required high professionalism, speed and accuracy along with physical 
health. At the same time, a healthy mental power must be supported to prevent secondary accidents that may occur during public service. In 2008, the study by the National Emergency Management Agency (formerly National Fire Service) found that over $80 \%$ of firefighters experienced traumatic events, while $35.9 \%$ said they needed mental health services.

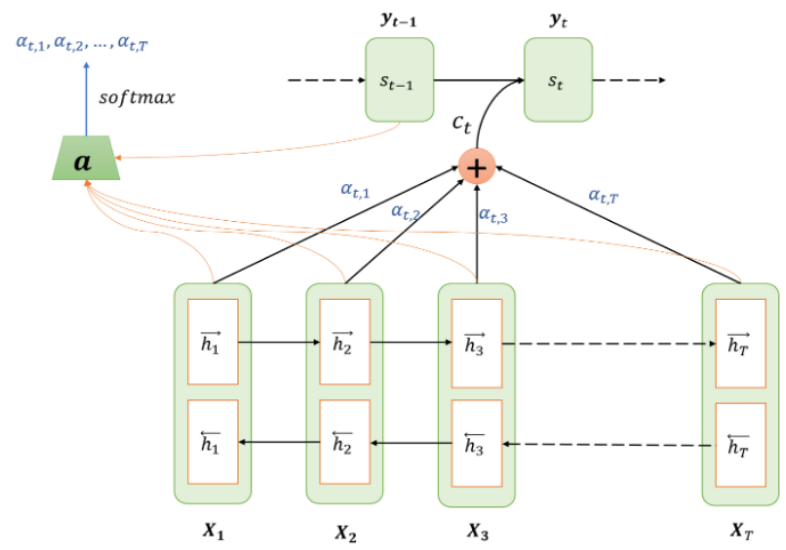

Figure 1. Example of using Keras and RNN analysis

Among the 2,705 fire department officials from 17 fire departments in Chungnam Province, fire emergency personnel were also selected as the study group. This paper also gathered the report and dispatch command data entered into the 119 Situation Room over the past two years. Through collecting survey data and deriving risk group standard data for rescue and paramedics, this paper defined data model of field activity personnel by dispatch order type. The research results are presented as follows.[8]

Table 1. Data model of fire field activity personnel by dispatch order type

\begin{tabular}{|c|c|c|c|c|c|c|c|c|c|}
\hline \multirow{2}{*}{ Classification } & \multicolumn{2}{|c|}{ PTSD } & \multicolumn{2}{c|}{$\begin{array}{c}\text { Problem with } \\
\text { Alcohol }\end{array}$} & \multicolumn{2}{c|}{$\begin{array}{c}\text { Depressive } \\
\text { Disorder }\end{array}$} & \multicolumn{2}{c|}{$\begin{array}{c}\text { Sleep } \\
\text { Disorder }\end{array}$} & $\begin{array}{c}\text { Intention } \\
\text { of care }\end{array}$ \\
\cline { 2 - 10 } & $\begin{array}{c}\text { Needed } \\
\text { Control }\end{array}$ & NeededCare & $\begin{array}{c}\text { Needed } \\
\text { Control }\end{array}$ & NeededCare & $\begin{array}{c}\text { Needed } \\
\text { Control }\end{array}$ & NeededCare & $\begin{array}{c}\text { Needed } \\
\text { Control }\end{array}$ & NeededCare & \\
\hline Number & 3,752 & 2,806 & 12,238 & 7,784 & 4,886 & 3,985 & 13,507 & 8,804 & 4,116 \\
\hline Ratio & $10.1 \%$ & $5.6 \%$ & $33 \%$ & $21 \%$ & $13.2 \%$ & $10.7 \%$ & $36.4 \%$ & $21.8 \%$ & $28.6 \%$ \\
\hline
\end{tabular}

Following is the analysis of research result. First, PTSD risk-inducing factors were analyzed by ICE-R, CESD, Suicide Accident (SBQ-R), and Alcohol Drinking Scale (AUDIT$\mathrm{K})$. Traumatic risk causing factors were analyzed according to the characteristics and types of field activities of the crew. Second, the actual rate of using the service was only $6.2 \%$, which was pointed out due to lack of substitutes and adjustment of working hours. At present, the lack of human and material resources for fire officials is unresolved, and it is also closely related to the recent issue of the transition to national positions of firefighters, who are highly interested in society. Firefighters currently classified as local officials are determined by the financial status of their local governments, which means that the survival rate of people in the event of disasters, and accidents is proportional to local finances. This is directly related to the treatment of firefighters, that is, the advancement of the working environment and health welfare. The central government support is urgently needed regardless of the phenomenon of 
the rich and poor. Third, there is a discrepancy in the quality of rescue and emergency services in case of emergency depending on the financial status of the region. Prompt, accurate rescue and first aid services for firefighters can be manifested from the healthy body and spirit, which can be found in the case of developed countries. The US fire department's top priority is "fireman's health and safety," and each state is enacting a law that provides mental health counseling and treatment services to families of firefighters, including firefighter suicide prevention programs. As a quantitative basis for such policy decision making, research on automatic analysis of risk groups and early warning system for big databased "traumatic stress disorder" is expected to be utilized as an important information asset for solving social problems. The number of PTSD patients in Korea is 7896, which is less than expected (Health Insurance Review and Assessment Service). This is because we do not know or hide that he/she is a patient. There is an atmosphere in Korea where it is difficult to talk about mental health at first, so the prevalence of mental disorders, including PTSD, is lower than in foreign countries. If we are constantly looking for information related to an infectious disease in anxiety, or if we are fleeing past places that create fear, we should be careful with PTSD. In particular, testing is recommended if he/she is in severe stress due to self-restraint among the isolates. Usually treatment works well within 4 weeks of the event. There are 'cognitive behavioral therapy' that reminds of trauma in a safe situation, and 'sensitization and reprocessing of eye movements' that maximizes the information processing function of the brain and blurs the memory [9][10].

\section{Conclusion}

This study collected the data from NEMA research focused on fire fighters activities containing high frequency of trauma and incident impact scale (IES-R), depression scale (CESD), suicide accident (SBQ-R), drinking scale (AUDIT-K). This paper analyzed the research data about the correlation with post-traumatic stress disorder triggering factors on the four scales. More concise and detail result of study can be collected by real-time dispatch types (fire, rescue, emergency) of report data and dispatch command data sent to the 119 situation room. And in the same way we can detects the risk of post-traumatic stress disorder and early warning of the field personnel dispatched to the report. As a quantitative basis for such policy decision making, research on automatic analysis of risk groups and early warning system for big data-based "traumatic stress disorder" is expected to be utilized as an important information asset for solving social problems.

\section{References}

[1] Bachlechner T., Majumder B. P., Mao H. H., Cottrell G. W., and McAuley J., "Rezero is all you need: Fast convergence at large depth," arXiv preprint arXiv:2003.04887, (2020)

[2] Brock A., Donahue J., and Simonyan K., "Large scale: GAN training for high fidelity natural image synthesis,” In International Conference on Learning Representations, (2019)

[3] Singh S. and Krishnan S., "Filter response normalization layer: Eliminating batch dependence in the training of deep neural networks," arXiv preprint arXiv:1911.09737, (2019)

[4] De S. and Smith S. L., "Batch: Normalization biases deep residual networks towards shallow paths," arXiv preprint arXiv:2002.10444, (2020)

[5] “Big Data Computing Technology," Hanbit Academy, pp.14-31, (2016)

[6] Real E., Liang C., So D. R., and Le Q. V., "Auto ML zero: Evolving machine learning algorithms from scratch,” arXiv preprint arXiv:2003.03384, (2020) 
[7] Zoph B. and Le Q. V., "Neural architecture search with reinforcement learning," In International Conference on Learning Representations, (2017)

[8] Real E., Aggarwal A., Huang Y., and Le Q. V., "Regularized evolution for image classifier architecture search," In Proceedings of the AAAI conference on artificial intelligence, vol.33, pp.4780-4789, (2019) DOI: 10.1609/aaai.v33i01.33014780

[9] Liu H., Simonyan K., Vinyals O., Fernando C., and Kavukcuoglu K., "Hierarchical: Representations for efficient architecture search," In International Conference on Learning Representations, (2018)

[10] Karras T., Aila T., Laine S., and Lehtinen J., "Progressive growing of gans for improved quality, stability, and variation,” In International Conference on Learning Representations, (2018) 